2

3

4

5

6

7

8

9

10

11

\title{
Effect of dead space on breathing stability at exercise in hypoxia.
}

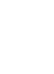

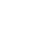

\section{Corresponding author}

richalet@univ-paris13.fr (J.P. Richalet)

eric.hermand17@gmail.com (E. Hermand)

Laboratoire “Hypoxie \& poumon”, EA2363

74 rue Marcel Cachin

93017 Bobigny Cedex, FRANCE

Tel: $+33148387758 /$ Fax: +33148388924

\section{Word count for manuscript: 2946}

Key words: Control of ventilation, hypoxia, exercise, periodic breathing, breathing instability, dead space, alveolar ventilation 
7

5

\section{Abstract}

Recent studies have shown that normal subjects exhibit periodic breathing when submitted to concomitant environmental (hypoxia) and physiological (exercise) stresses. A mathematical model including mass balance equations confirmed the short period of ventilatory oscillations and pointed out an important role of dead space in the genesis of these phenomena. Ten healthy subjects performed mild exercise on a cycloergometer in different conditions: rest/exercise, normoxia/hypoxia and no added dead space / added dead space (aDS). Ventilatory oscillations (V்e peak power) were augmented by exercise, hypoxia and aDS $(\mathrm{P}<0.001, \mathrm{P}<0.001$ and $\mathrm{P}<0.01$, respectively) whereas $\dot{\mathrm{V} E}$ period was only shortened by exercise $(\mathrm{P}<0.001)$, with an 11 -second period. aDS also increased $\dot{\mathrm{VE}}(\mathrm{P}<0.001)$, tidal volume (VT, $\mathrm{P}<0.001)$, and slightly augmented $\mathrm{PETCO}_{2}(\mathrm{P}<0.05)$ and the respiratory frequency $(\mathrm{P}<0.05)$. These results confirmed our previous model, showing an exacerbation of breathing instability by increasing dead space. This underlines opposite effects observed in heart failure patients and normal subjects, in which added dead space drastically reduced periodic breathing and sleep apneas. It also points out that alveolar ventilation remains very close to metabolic needs and is not affected by an added dead space.

Clinical Trial reg. $\mathrm{n}^{\circ}$ : NCT02201875 


\section{Introduction}

Since the $19^{\text {th }}$ century and its first description by Drs Cheyne and Stokes (Naughton, 1998), periodic breathing has been the center of numerous studies on ventilatory stability among normal subjects and patients. This dysfunction of respiration control is mainly observed in patients suffering from chronic heart failure (CHF) (Javaheri, 1999) or sleep apneas, from central (Javaheri and Dempsey, 2013) (SACS), mixed or obstructive (Pham and Schwartz, 2015) (SAOS) origin, but also in healthy subjects at high altitude, mostly in sleep state (Ainslie et al., 2013). The pathophysiology of these manifestations involves a decreased cardiac output ( $\dot{\mathrm{Q}}$ ) (Agostoni et al., 2015) and/or a hypersensitivity of peripheral and central chemoreceptors to $\mathrm{O}_{2}$ and $\mathrm{CO}_{2}$ stimuli (Chua et al., 1996; Francis et al., 2000; Giannoni et al., 2008; Ponikowski et al., 2001; Solin et al., 2000). However, recent studies (Hermand et al., 2015b, 2015a; Latshang et al., 2013) have paradoxically underlined a similar phenomenon in normal subjects submitted to concomitant metabolic (exercise) and environmental (hypoxic) stresses. In these conditions, the amplitude of periodic breathing is positively related to both $\dot{\mathrm{Q}} \mathrm{c}$ and ventilation ( $\dot{\mathrm{V} E}$ ), as opposed to the observations in CHF and SACS/SAOS patients whereas its period, much shorter (between 11 and 13 seconds), is inversely related to $\dot{Q} c$ and $\dot{\mathrm{VE}}$.

Several methods to treat periodic breathing and sleep apneas are being used or experimented, with a variable efficiency: CPAP and oxygen therapy (Pearse and Cowie, 2016), acetazolamide (Javaheri, 2006; White et al., 1982) and $\mathrm{CO}_{2}$ inhalation (Szollosi et al., 2004; Wan et al., 2013). An alternative approach is the use of an additional external dead space, which has proven to be effective by drastically reducing periodic breathing during in patients (Khayat et al., 2003; Sharma et al., 2011; Xie et al., 1997) and in subjects at high altitude (Lovis et al., 2012; Patz et al., 2013). By maintaining a relatively high arterial partial pressure in $\mathrm{CO}_{2}\left(\mathrm{PaCO}_{2}\right)$ combined with a higher $\dot{\mathrm{V}}$, this device prevents its fall below the apnea threshold and the consequent genesis of a central apnea cycle. Augmented dead space also alters the breathing pattern when increasing $\dot{V} E$, as respiratory rate remains stable while tidal volume (VT) augments (Agostoni et al., 2008; Khayat et al., 2003; Xie et al., 1997). This underlines the additional contribution of low tidal volume in central sleep apneas in patients, besides cardiac output and chemosensitivity.

In exercise conditions, scarce studies highlight a similar inhibiting effect of added dead space on periodic breathing in CHF patients (Agostoni et al., 2008).

However, in a previous work, we developed a model in order to evaluate the respective influence of various factors on breathing stability during moderate exercise in hypoxia. Our 
model predicted that a decrease in alveolar ventilation / minute ventilation ratio (i்/VंE), therefore an increase in dead space, would increase breathing instability and ventilatory oscillations (Hermand et al., 2016), regardless of cardiac output and chemosensitivity. Therefore, we aimed to validate our mathematical model in normal subjects at moderate exercise in hypoxia, by adding dead space in the breathing circuit and we hypothesized that this intervention would promote breathing instability.

\section{Subjects and methods}

\section{Subjects.}

Ten healthy non-smoking male subjects volunteered for the study and were given complete information about the successive tests. All were in good physical condition, with a medium to high level of regular physical activity (from 2 to 10 hours per week). A preliminary medical examination showed no evidence of cardiovascular or pulmonary disease. Subjects' characteristics are presented in table 1. The protocol was approved by the Ile-de-France Ethics Committee (CPP-IDF2) and an individual written informed consent has been collected from all subjects. The study was registered as Clinical Trial reg. $\mathrm{n}^{\circ}$ : NCT02201875.

\section{Procedure.}

All subjects were first asked to perform a standard ramp test protocol on a cycloergometer to be familiarized with calibrated breathing apparatus and determine their maximal aerobic power (MAP, Table 1): after a 3-minute warm-up at 60 watts, power output was increased by 30 -watt steps every two minutes until exhaustion.

Four tests were then randomly conducted, in normoxia and hypoxia (fraction of inspired $\mathrm{O}_{2}, \mathrm{FIO}_{2}=14,5 \%$ ), in accordance with previous work (Hermand et al., 2015a), with and without a $500 \mathrm{~mL}$ added dead space (aDS), consisting in a cylindrical tube (diameter $5 \mathrm{~cm}$ ) clipped on the mouthpiece. This volume was determined accordingly with our predictive model (Hermand et al., 2016) and with the existing literature (Khayat et al., 2003; Lovis et al., 2012; Patz et al., 2013).

After one minute rest for material habituation and cardiorespiratory parameters stabilization, subjects were first asked to keep a resting sitting position on the ergometer for 6 min, and then to pedal for $6 \mathrm{~min}$ at around $65 \mathrm{rpm}$ pedaling cadence at an exercise intensity of $30 \%$ of MAP (Hermand et al., 2015a). 
$\dot{\mathrm{VE}}\left(\mathrm{L} \cdot \mathrm{min}^{-1}\right)$ and heart rate (HR, bpm) were measured through a metabograph (Vmax Encore, SensorMedics, Yorba Linda, CA). VT (L), $\mathrm{O}_{2}$ consumption $\left(\mathrm{V}_{2}, \mathrm{~L}_{\mathrm{min}}{ }^{-1}\right), \mathrm{CO}_{2}$ production $\left(\dot{\mathrm{VCO}}_{2}, \mathrm{~L} \cdot \mathrm{min}^{-1}\right)$, total respiratory cycle time (Ttot, s) and inspiratory time (Ti, s) were derived from the ventilation signal. Pulse $\mathrm{O}_{2}$ saturation $\left(\mathrm{SpO}_{2}, \%\right)$ was measured by transcutaneous oximetry (Nellcor N-595, Nellcor, Pleasanton, CA) on a pre-warmed ear lobe. End tidal $\mathrm{PCO}_{2}\left(\mathrm{PETCO}_{2}, \mathrm{mmHg}\right)$ was measured by infrared thermopile (Vmax Encore, SensorMedics, Yorba Linda, CA). During the whole test, $\dot{\mathrm{VE}}, \mathrm{SpO}_{2}$ and $\mathrm{PETCO}_{2}$ were recorded breath-by-breath (Fig. 1). Data were transferred to a computer for further variability analysis. A Fast Fourier Transform (FFT) was then applied to the breath-by-breath ventilation signal, extracted from the raw data, in sequences of 128 points (one point per second) of a steady state interval at the end of each phase of the test. This method allowed us to detect the presence of peaks in the frequency domain of the ventilation signal (Fig. 2) (Hermand et al., 2015b). Two main parameters were derived from the FFT: the frequency in hertz (or period in seconds) of the larger peak and its power estimated as the area under the peak at $\pm 0.02 \mathrm{~Hz}$ around the peak (in $\mathrm{L}^{2} \cdot \mathrm{min}^{-2}$ for $\dot{\mathrm{V} E}$ spectrum). Thus, a high peak power translates into greater ventilatory oscillations. This method allowed us to precisely quantify the presence of oscillations in the signals that are not observable in a standard protocol routinely used where the signals are averaged every 20 seconds (Bourdillon et al., 2014; Canouï-Poitrine et al., 2014; Lhuissier et al., 2012; Richalet et al., 2012). Finally, alveolar ventilation was assessed from the values of end tidal $\mathrm{CO}_{2}$ pressure, as a surrogate of alveolar $\mathrm{CO}_{2}$ pressure $\left(\mathrm{P}_{\mathrm{A}} \mathrm{CO}_{2}\right)$ and $\mathrm{CO}_{2}$ production rate $(\dot{\mathrm{V} C O})_{2}$, which anatomical dead space (VD), alveolar / total ventilation ratio ( $\dot{\mathrm{V} A} / \mathrm{VE}$ ) and dead space / tidal (VD/VT) are derived from (Whipp and Ward, 1998):

$$
\dot{V} A=\frac{\dot{V} C O_{2}}{K \cdot P_{A} C O_{2}}
$$

where $\mathrm{K}$ is the constant to adapt standard temperature and pressure dry (STPD) to body temperature pressure and saturation (BTPS) conditions;

$$
V D=\frac{(\dot{V} E-\dot{V} A)}{F R}
$$

where FR is the respiratory frequency $\left(\mathrm{min}^{-1}\right)$

Statistical analysis. Results are presented as mean \pm standard deviation. Regarding the expected difference in mean period ( 3 seconds), the standard deviation ( 2.4 seconds), a level of significance at $0.05 \%$ and a statistical power at $80 \%$, the minimum number of subjects to 
be included was 7 for each protocol. Since spectral peak power showed a high standard deviation and a non-normal distribution, we performed a logarithmic transformation of raw data, and calculated a minimum number of subjects of 10 to include. Normality of data on each condition (rest/exercise, normoxia/hypoxia, without/with aDS) was verified by a Shapiro-Wilk normality test. Thus, a three-way analysis of variance (ANOVA) with repeated measures were done, including interactions between factors. A post-hoc paired Student's test was used when applicable. As exercise is associated with a concomitant variation of multiple variables, we performed a multivariate regression analysis in order to evaluate the independent influence of each cardiorespiratory variable on period and peak power of $\dot{\mathrm{V} E}$ spectrum. This approach, for example, will allow us to assess the specific influence of $\dot{V} E$ and/or $\dot{Q} c$ on peak power and period of $\dot{V} E$.

\section{Results}

Values of measured cardiorespiratory variables and post-hoc tests in subgroups are presented in table 2 and in figures 3, 4, 5 and 6.

\section{Effect of exercise vs rest}

As expected, $\dot{\mathrm{VE}}, \dot{\mathrm{V}} \mathrm{O}_{2}, \dot{\mathrm{V}} \mathrm{CO}_{2}, \mathrm{PETCO}_{2}, \mathrm{VT}, \mathrm{HR}, \dot{\mathrm{V} A}$ and $\dot{\mathrm{V}} / \mathrm{V} \mathrm{V}$ increased with exercise $(P<$ $0.001)$ whereas $\mathrm{SpO}_{2}$, Ttot and VD/VT decreased $(P<0.001)$. VE peak power was greater $(P$ $<0.001)$ and its period shorter $(P<0.001)$.

In hypoxia, $\mathrm{O}_{2}$ desaturation was accentuated by exercise for both $\mathrm{aDS}$ condition $(\mathrm{P}<0.01)$.

\section{Effect of hypoxia vs normoxia}

Hypoxia increased $\dot{\mathrm{VE}}(P<0.01), \mathrm{HR}, \dot{\mathrm{V} A}(P<0.001), \dot{\mathrm{VA}}, \dot{\mathrm{VA}} / \dot{\mathrm{VE}}(\mathrm{P}<0.05)$ and decreased $\mathrm{SpO}_{2}$ and $\mathrm{PETCO}_{2}(P<0.001)$ and VD/VT $(\mathrm{P}<0.05)$ whereas Ttot, VD and VT were not modified, except a trend for a rise in VT $(P=0.06)$. $\dot{\mathrm{VE}}$ peak power was augmented by hypoxia $(P<0.001)$ and period of $\dot{\mathrm{V} E}$ oscillations remained unchanged.

During exercise, $\dot{\mathrm{V} E}$ was not modified by hypoxia at $\mathrm{aDS}=0$ but significantly increased with a $500 \mathrm{~mL}$ aDS $(\mathrm{P}<0.001)$.

\section{Effect of added dead space}

$\dot{\mathrm{VE}}$, VT and $\dot{\mathrm{VO}}_{2}$ increased with the aDS $(P<0.001)$ whereas Ttot was shortened $(P<0.05)$. $\mathrm{PETCO}_{2}$ was very slightly increased $(+1.8 \%, \mathrm{P}<0.05)$. Added dead space had no effect on 
$\mathrm{SpO}_{2}$ and $\dot{\mathrm{V}}$. However, it enhanced $\dot{\mathrm{VE}}$ peak power $(P<0.001)$, period remaining unchanged.

In most conditions, despite large increases in $\dot{V E}$ due to aDS (+ $42 \%$, all values pooled, fig. 4 , upper left), $\dot{\mathrm{V} A}$ remains stable and is not affected by aDS (Fig. 4, upper right).

In hypoxia, aDS increased $\dot{\mathrm{V} A}$ and $\dot{\mathrm{V} E}$ peak power during exercise in hypoxia $(\mathrm{P}<0.05, \mathrm{P}<$ 0.01 and respectively, fig. 4 and 5).

\section{Interactions between factors}

The ANOVA showed that exercise accentuated, respectively, the effect of aDS on $\dot{\mathrm{VE}}, \dot{\mathrm{V}} \mathrm{O}_{2}$ and on $\dot{V} A / \dot{V} E$, the effect of hypoxia on $\mathrm{O}_{2}$ desaturation, the effect of hypoxia on decreased $\mathrm{PETCO}_{2}$, the effect of aDS on increased $\dot{\mathrm{VE}}$ peak power.

\section{Multivariate regression analysis: factors influencing $\dot{V} E$ peak power and period}

Among various cardiorespiratory parameters, $\dot{\mathrm{V} E}$ peak power was positively correlated to $\dot{\mathrm{V} E}$ and $\mathrm{PETCO}_{2}$ (Fig. 6, $\mathrm{P}<0.001$ ), with a trend for $\dot{\mathrm{VA}} / \dot{\mathrm{V} E}(\mathrm{r}=0.67, \mathrm{P}=0.073)$. $\mathrm{V} E$ period was strongly positively correlated to Ttot (Fig. 6, P < 0.001) and, in a lesser extent, negatively correlated to $\dot{\mathrm{V}} \mathrm{A} / \mathrm{V} E(\mathrm{P}<0.01)$.

\section{Discussion}

Two main conclusions might be drawn from these original data. First, this study confirms the mathematical simulations of the impact of added dead space on ventilatory instability in healthy subjects, during exercise in hypoxia (Fig. 1). Second, unlike the observations made in CHF/SAS patients and normal subjects during sleep at high altitude, an increased dead space promotes respiratory instability and ventilatory oscillations.

As observed in patients and normal subjects (Ainslie et al., 2013; Khayat et al., 2003), the breathing pattern with aDS was mainly affected by VT swings (Fig. 3, lower panels). In addition, respiratory frequency was equally shortened by added dead space at rest and exercise $(-8 \%$ and $-7 \%$, respectively): as the added dead space induces a higher alveolar $\mathrm{PCO}_{2}$, the ventilatory control system in a normal subject quickly adjusts $\dot{\mathrm{V} E}$ to eliminate $\mathrm{CO}_{2}$ by augmenting both VT and respiratory rate, although in a lesser extent, as opposed to what is observed in CHF patients (Khayat et al., 2003). This illustrates a fully functional respiratory 
output in a normal subject. On a side note, the slight metabolic increase of $\mathrm{V}_{2}$ with aDS ( $+9 \%$, all conditions, fig. 5), along with a similar trend for $\dot{\mathrm{V}} \mathrm{CO}_{2}(\mathrm{P}=0.075)$, could be attributed to the additional workload of respiratory muscles (Lorenzo and Babb, 2012), due to a substantial increase of $\dot{V} E$, mainly through a large rise in VT. However, the increase of $\dot{V} E$ did not modify $\mathrm{SpO}_{2}$ as it does in patients and normal subjects at high altitude (Khayat et al., 2003; Lovis et al., 2012; Xie et al., 1997) (Fig. 3).

This study confirms that the ventilatory control is destabilized when the system is submitted to a double concomitant, physiological and environmental, stress, at exercise in hypoxia (Hermand et al., 2015b, 2015a). Thus, amplitude of ventilatory oscillations are correlated to ventilation level and cardiac output (not shown) and their period, inferior to 11 seconds at exercise and therefore shorter than those observed in CHF and SAS patients (Francis et al., 2000)(Fig. 2), is strongly correlated to Ttot (Fig. 6).

This striking difference may have an origin in the regulatory system of arterial $\mathrm{CO}_{2}$ pressure. A well-known mechanism preventing a CHF/SAS patient from starting an apnea/hyperpnea cycle is to keep $\mathrm{PaCO}_{2}$ above an apnea threshold (Xie et al., 2002) using $\mathrm{CO}_{2}$ inhalation or external dead space device, even though the ventilation is augmented by the respiratory command via a central $\mathrm{CO}_{2}$ chemoreflex activation. This explains why, in this context, the fall of apnea/hypopnea index (AHI) is systematically associated with a large increase in $\mathrm{PaCO}_{2}$ and an inadequate response to hypercapnia due to an augmented ventilatory response to $\mathrm{CO}_{2}$, leading to successive $\mathrm{CO}_{2}$-threshold crossings and apnea cycles (Khayat et al., 2003; Xie et al., 1997). The opposite phenomenon takes place in normal subjects: a slight change in arterial $\mathrm{PCO}_{2}$ induces an immediate and appropriate ventilatory response to bring the system back to a homeostatic equilibrium. For instance, in CHF patients at rest, a $\sim 15 \%$ rise in $\mathrm{PETCO}_{2}$, from 37 to $42 \mathrm{mmHg}$, elicits a $~ 50 \%$ increase in VंE, from 6.4 to $9.6 \mathrm{~L}^{\mathrm{min}}{ }^{-1}$ (Khayat et al., 2003) whereas a mere $3 \% \mathrm{PETCO}_{2}$ gain, from 37.9 to $39.1 \mathrm{mmHg}$, in healthy subjects induces a $67 \%$ rise in $\dot{\mathrm{VE}}$, from 11.1 to $18.5 \mathrm{~L} \cdot \mathrm{min}^{-1}$ (the $\dot{\mathrm{VE}}$ response is even greater at exercise). In the latter, a well-functioning $\mathrm{CO}_{2}$ control system limits the $\mathrm{aDS}$-induced $\mathrm{CO}_{2}$ rise to a very low range, but nevertheless drastically augments the ventilation level which is associated to increased breathing instability at exercise (Hermand et al., 2015a, 2015b) (Fig. 5). In that perspective, the same remarkable mechanism was recently pointed out in obese patients with and without obstructive SAOS, in which progressive aDS-induced variations of $\mathrm{PETCO}_{2}$ were kept within a $2 \mathrm{mmHg}$ margin by rises of $\dot{V} E$ (Bernhardt et al., 2017).

It is interesting to note that the oscillatory pattern enhanced by adding dead space might be explained by the difference between the input and the output of the control system: the values 
of blood gases $\left(\mathrm{PaO}_{2}\right.$ and $\left.\mathrm{PaCO}_{2}\right)$ are determined by alveolar ventilation ( $\left.\dot{\mathrm{VA}}\right)$, while these blood gases act on total ventilation ( $\dot{\mathrm{VE}})$ via the chemoreceptors and control centers. Thus, any difference between $\dot{V} A$ and $\dot{V} E$ will promote instability in blood gases and therefore in ventilation.

These systemic properties unveiled a second and noticeable mechanism: a stable VंA despite large swings of $\dot{\mathrm{V} E}$ in most conditions underlines a remarkable adaptation of ventilatory control system, in order to maintain the "effective" respiration, $\mathrm{VA}$, close to the metabolic needs of the organism, under environmental and physiological stresses, regarding the dead space status (VD, fig. 4, lower right). Assuming that the ratio ventilation-perfusion is optimal in healthy subjects at rest and at mild exercise (Moinard et al., 2004), we suggest that the "extra" ventilation with aDS (Fig. 3 and 4) is then attributed to the sole action of $\mathrm{CO}_{2}$ chemoreflex, combined from peripheral and central chemoreceptors.

Central sleep apneas exhibited by normal subjects at high altitude are also greatly reduced by added dead space (Lovis et al., 2012; Patz et al., 2013). However, underlying mechanisms are different from patients. A broad consensus point out that an augmented activity of peripheral chemoreceptors in hypoxia is involved into the genesis of periodic breathing (Ainslie et al., 2013; Hermand et al., 2015b; Kumar and Prabhakar, 2012; Lahiri et al., 2006), in a subtle interplay with central chemoreceptors through peripheral afferences into the nucleus tractus solitarii (NTS) (Smith et al., 2013). A higher VंE by aDS-induced central $\mathrm{CO}_{2}$ stimulation will maintain a higher $\mathrm{O}_{2}$ saturation (Patz et al., 2013), lowering carotid bodies activity and its subsequent effect on periodic breathing. In the present work, aDS did not enhance $\mathrm{SpO}_{2}$ in spite of a large increase in $\dot{V} E$ especially in hypoxia, maintaining a higher peripheral sensitivity and therefore a greater tendency to develop periodic breathing. This may explain the oscillatory exacerbation observed in hypoxia, even more at exercise, during which $\mathrm{O}_{2}$ desaturation is worsened. Furthermore, the prompt response of ventilatory control system to a higher aDS-induced $\mathrm{PaCO}_{2}$ within a narrow range points out the role of $\mathrm{CO}_{2}$ chemosensitivity, enhanced in hypoxia (Masuyama et al., 1989), leading to greater ventilatory oscillations than those observed without added dead space. Whereas the latter points require further studies to quantify the involvement of central and peripheral chemoreceptors in this matter, other potential actors could come into play, such as the action of lung stretch receptors which could be activated by an augmented VT with aDS.

Lastly, we confirmed the tight relationship existing between VE period and Ttot (Hermand et al., 2015a, 2015b) (Fig. 6, right), suggesting the existence of an intrinsic oscillator modulating the activity of the central pattern generator (Forster et al., 2014). The theoretical limit of $\dot{V} E$ 
period, when Ttot tends towards zero (i.e. respiratory frequency is infinite), is around 6 seconds.

In conclusion, these findings confirm the destabilizing role of added dead space in the ventilatory control system of normal subjects at exercise in hypoxia, as anticipated by a mathematical model. It also points out the discrepancy of the effect of $\mathrm{CO}_{2}$ between healthy subjects on one hand, and CHF/SAS patients and normal subjects asleep in altitude on the other hand, counteracting or promoting breathing stability. This study, in an original manner, confirmed that total ventilation adapts to external constraints by maintaining adequate alveolar ventilation for metabolic needs of the organism.

\section{References}

Agostoni, P., Apostolo, A., Albert, R.K., 2008. Mechanisms of periodic breathing during exercise in patients with chronic heart failure. Chest 133, 197-203. doi:10.1378/chest.07-1439

Agostoni, P., Contini, M., Vignati, C., Del Torto, A., De Vecchi Lajolo, G., Salvioni, E., Spadafora, E., Lombardi, C., Gerosa, G., Bottio, T., Morosin, M., Tarzia, V., Scuri, S., Parati, G., Apostolo, A., 2015. Acute Increase of Cardiac Output Reduces Central Sleep Apneas in Heart Failure Patients. J. Am. Coll. Cardiol. 66, 2571-2572. doi:10.1016/j.jacc.2015.09.074

Ainslie, P.N., Lucas, S.J.E., Burgess, K.R., 2013. Breathing and sleep at high altitude. Respir Physiol Neurobiol 188, 233-256. doi:10.1016/j.resp.2013.05.020

Bernhardt, V., Mitchell, G.S., Lee, W.Y., Babb, T.G., 2017. Short-term modulation of the ventilatory response to exercise is preserved in obstructive sleep apnea. Respir Physiol Neurobiol 236, 42-50. doi:10.1016/j.resp.2016.11.003

Bourdillon, N., Fan, J.-L., Kayser, B., 2014. Cerebral oxygenation during the Richalet hypoxia sensitivity test and cycling time-trial performance in severe hypoxia. Eur. J. Appl. Physiol. 114, 10371048. doi:10.1007/s00421-014-2835-8

Canouï-Poitrine, F., Veerabudun, K., Larmignat, P., Letournel, M., Bastuji-Garin, S., Richalet, J.-P., 2014. Risk prediction score for severe high altitude illness: a cohort study. PLoS ONE 9, e100642. doi:10.1371/journal.pone.0100642

Chua, T.P., Clark, A.L., Amadi, A.A., Coats, A.J., 1996. Relation between chemosensitivity and the ventilatory response to exercise in chronic heart failure. J. Am. Coll. Cardiol. 27, 650-657.

Forster, H., Bonis, J., Krause, K., Wenninger, J., Neumueller, S., Hodges, M., Pan, L., 2014. Contributions of the pre-Bötzinger complex and the Kölliker-fuse nuclei to respiratory rhythm and pattern generation in awake and sleeping goats. Prog. Brain Res. 209, 73-89. doi:10.1016/B978-0444-63274-6.00005-9

Francis, D.P., Willson, K., Davies, L.C., Coats, A.J., Piepoli, M., 2000. Quantitative general theory for periodic breathing in chronic heart failure and its clinical implications. Circulation 102, 2214-2221. 
Giannoni, A., Emdin, M., Poletti, R., Bramanti, F., Prontera, C., Piepoli, M., Passino, C., 2008. Clinical significance of chemosensitivity in chronic heart failure: influence on neurohormonal derangement, Cheyne-Stokes respiration and arrhythmias. Clin. Sci. 114, 489-497. doi:10.1042/CS20070292

Hermand, E., Lhuissier, F.J., Larribaut, J., Pichon, A., Richalet, J.-P., 2015a. Ventilatory oscillations at exercise: effects of hyperoxia, hypercapnia, and acetazolamide. Physiol Rep 3. doi:10.14814/phy2.12446

Hermand, E., Lhuissier, F.J., Voituron, N., Richalet, J.-P., 2016. Ventilatory oscillations at exercise in hypoxia: A mathematical model. Journal of Theoretical Biology 411, 92-101. doi:10.1016/j.jtbi.2016.10.002

Hermand, E., Pichon, A., Lhuissier, F.J., Richalet, J.-P., 2015b. Periodic breathing in healthy humans at exercise in hypoxia. J. Appl. Physiol. 118, 115-123. doi:10.1152/japplphysiol.00832.2014

Javaheri, S., 2006. Acetazolamide improves central sleep apnea in heart failure: a double-blind, prospective study. Am. J. Respir. Crit. Care Med. 173, 234-237. doi:10.1164/rccm.200507-1035OC Javaheri, S., 1999. A mechanism of central sleep apnea in patients with heart failure. N. Engl. J. Med. 341, 949-954. doi:10.1056/NEJM199909233411304

Javaheri, S., Dempsey, J.A., 2013. Central sleep apnea. Compr Physiol 3, 141-163. doi:10.1002/cphy.c110057

Khayat, R.N., Xie, A., Patel, A.K., Kaminski, A., Skatrud, J.B., 2003. Cardiorespiratory Effects of Added Dead Space in Patients With Heart Failure and Central Sleep Apnea. Chest 123, 1551-1560. doi:10.1378/chest.123.5.1551

Kumar, P., Prabhakar, N.R., 2012. Peripheral chemoreceptors: Function and plasticity of the carotid body, in: Terjung, R. (Ed.), Comprehensive Physiology. John Wiley \& Sons, Inc.

Lahiri, S., Roy, A., Baby, S.M., Hoshi, T., Semenza, G.L., Prabhakar, N.R., 2006. Oxygen sensing in the body. Prog. Biophys. Mol. Biol. 91, 249-286. doi:10.1016/j.pbiomolbio.2005.07.001

Latshang, T.D., Turk, A.J., Hess, T., Schoch, O.D., Bosch, M.M., Barthelmes, D., Merz, T.M., Hefti, U., Hefti, J.P., Maggiorini, M., Bloch, K.E., 2013. Acclimatization improves submaximal exercise economy at $5533 \mathrm{~m}$. Scand J Med Sci Sports 23, 458-467. doi:10.1111/j.1600-0838.2011.01403.x

Lhuissier, F.J., Brumm, M., Ramier, D., Richalet, J.-P., 2012. Ventilatory and cardiac responses to hypoxia at submaximal exercise are independent of altitude and exercise intensity. J. Appl. Physiol. 112, 566-570. doi:10.1152/japplphysiol.00906.2011

Lorenzo, S., Babb, T.G., 2012. Oxygen cost of breathing and breathlessness during exercise in nonobese women and men. Med Sci Sports Exerc 44, 1043-1048. doi:10.1249/MSS.0b013e3182444c4b

Lovis, A., De Riedmatten, M., Greiner, D., Lecciso, G., Andries, D., Scherrer, U., Wellman, A., Sartori, C., Heinzer, R., 2012. Effect of added dead space on sleep disordered breathing at high altitude. Sleep Med. 13, 663-667. doi:10.1016/j.sleep.2012.02.012

Masuyama, S., Kohchiyama, S., Shinozaki, T., Okita, S., Kunitomo, F., Tojima, H., Kimura, H., Kuriyama, T., Honda, Y., 1989. Periodic breathing at high altitude and ventilatory responses to O2 and 
361

Moinard, J., Yquel, R., Manier, G., 2004. Pulmonary gas exchange during exercise in healthy subjects. Rev Mal Respir 21, 950-960.

Naughton, M.T., 1998. Pathophysiology and treatment of Cheyne-Stokes respiration. Thorax 53, 514518. doi:10.1136/thx.53.6.514

Patz, D.S., Patz, M.D., Hackett, P.H., 2013. Dead space mask eliminates central apnea at altitude. High Alt. Med. Biol. 14, 168-174. doi:10.1089/ham.2012.1111

Pearse, S.G., Cowie, M.R., 2016. Sleep-disordered breathing in heart failure. Eur. J. Heart Fail. doi:10.1002/ejhf.492

Pham, L.V., Schwartz, A.R., 2015. The pathogenesis of obstructive sleep apnea. J Thorac Dis 7, 13581372. doi:10.3978/j.issn.2072-1439.2015.07.28

Ponikowski, P., Chua, T.P., Anker, S.D., Francis, D.P., Doehner, W., Banasiak, W., Poole-Wilson, P.A., Piepoli, M.F., Coats, A.J., 2001. Peripheral chemoreceptor hypersensitivity: an ominous sign in patients with chronic heart failure. Circulation 104, 544-549.

Richalet, J.-P., Larmignat, P., Poitrine, E., Letournel, M., Canouï-Poitrine, F., 2012. Physiological risk factors for severe high-altitude illness: a prospective cohort study. Am. J. Respir. Crit. Care Med. 185, 192-198. doi:10.1164/rccm.201108-1396OC

Sharma, B., McSharry, D., Malhotra, A., 2011. Sleep disordered breathing in patients with heart failure: pathophysiology and management. Curr Treat Options Cardiovasc Med 13, 506-516. doi:10.1007/s11936-011-0145-6

Smith, J.C., Abdala, A.P.L., Borgmann, A., Rybak, I.A., Paton, J.F.R., 2013. Brainstem respiratory networks: building blocks and microcircuits. Trends Neurosci. 36, 152-162. doi:10.1016/j.tins.2012.11.004

Solin, P., Roebuck, T., Johns, D.P., Walters, E.H., Naughton, M.T., 2000. Peripheral and central ventilatory responses in central sleep apnea with and without congestive heart failure. Am. J. Respir. Crit. Care Med. 162, 2194-2200. doi:10.1164/ajrccm.162.6.2002024

Szollosi, I., Jones, M., Morrell, M.J., Helfet, K., Coats, A.J.S., Simonds, A.K., 2004. Effect of CO2 inhalation on central sleep apnea and arousals from sleep. Respiration 71, 493-498. doi:10.1159/000080634

Wan, Z.H., Wen, F.J., Hu, K., 2013. Dynamic $\mathrm{CO}_{2}$ inhalation: a novel treatment for CSR-CSA associated with CHF. Sleep Breath 17, 487-493. doi:10.1007/s11325-012-0719-x

Whipp, B.J., Ward, S.A., 1998. Determinants and control of breathing during muscular exercise. Br J Sports Med 32, 199-211.

White, D.P., Zwillich, C.W., Pickett, C.K., Douglas, N.J., Findley, L.J., Weil, J.V., 1982. Central sleep apnea. Improvement with acetazolamide therapy. Arch. Intern. Med. 142, 1816-1819.

Xie, A., Rankin, F., Rutherford, R., Bradley, T.D., 1997. Effects of inhaled CO2 and added dead space on idiopathic central sleep apnea. J. Appl. Physiol. 82, 918-926.

Xie, A., Skatrud, J.B., Puleo, D.S., Rahko, P.S., Dempsey, J.A., 2002. Apnea-hypopnea threshold for 
$398 \mathrm{CO} 2$ in patients with congestive heart failure. Am. J. Respir. Crit. Care Med. 165, 1245-1250.

399 doi:10.1164/rccm.200110-022OC

400

401 


\section{CAPTIONS}

Figure 1. Example of breath-by-breath ventilation recordings in hypoxia, without and with added dead space (respectively upper and lower panels). Ventilatory instability is noticeably increased with added dead space.

Figure 2. Means of power spectral densities of the ventilation signal at exercise in 3 conditions: normoxia and hypoxia without added dead space (aDS), hypoxia with aDS (plain, dot and dashed lines, respectively). Peak power is higher when aDS is combined to both exercise and hypoxia.

Figure 3. Mean $( \pm \mathrm{SD})$ values of $\mathrm{O}_{2}$ saturation $\left(\mathrm{SpO}_{2}\right)$, end-tidal $\mathrm{CO}_{2}$ pressure $\left(\mathrm{PETCO}_{2}\right)$, tidal volume (VT) and respiratory frequency. Exercise vs Rest: \#, $P<0.05$; \#\#, $P<0.01$; \#\#, $P<0.001$. Hypoxia vs normoxia: * $P<0.05$; **, $P<0.01$; ***, $P<0.001$. aDS 500 vs aDS $0: \dagger, P<0.05 ; \dagger \dagger, P<0.01 ; \dagger \dagger \uparrow, P<0.001$

Figure 4. Mean $( \pm \mathrm{SD})$ values of minute and alveolar ventilation ( $\dot{\mathrm{V} E}$ and $\dot{\mathrm{V}} \mathrm{A}$ respectively), the $\dot{\mathrm{V}} / \dot{\mathrm{V}} \mathrm{E}$ ratio and the assessed total dead space (VD). Exercise vs Rest: \#, $P<0.05$; \#\#, $P<$ 0.01; \#\#\#, $P<0.001$. Hypoxia vs normoxia: * $P<0.05$; **, $P<0.01$; ***,$P<0.001$. aDS 500 vs aDS $0: \dagger, P<0.05 ; \dagger \dagger, P<0.01 ; \dagger \dagger \dagger, P<0.001$

Figure 5. Ventilation peak power ( $\dot{\mathrm{V} E}$ peak power, left) and period (i் period, right). Exercise vs Rest: \#, $P<0.05$; \#\#, $P<0.01$; \#\#\#, $P<0.001$. Hypoxia vs normoxia: $*, P<$ 0.05 ; **, $P<0.01$; ***, $P<0.001$. Exercise vs Rest: \#, $P<0.05$; \#\#, $P<0.01$; \#\#\#, $P<$ 0.001. Hypoxia vs normoxia: *,$P<0.05$; ** $P<0.01$; ***, $P<0.001$ aDS 500 vs aDS $0: \dagger$, $P<0.05 ; \dagger \dagger, P<0.01 ; \dagger \dagger, P<0.001$

Figure 6. $\dot{V} E$ peak power (left) and period (right) as a function of $\dot{V} E$ and Ttot, respectively. Rest and exercise values have been pooled in all conditions. 
Table 1. Characteristics of the subjects

\begin{tabular}{lccccc}
\hline & n & $\begin{array}{c}\text { Age } \\
(\mathrm{yrs})\end{array}$ & $\begin{array}{c}\text { Body } \\
\text { weight } \\
(\mathrm{kg})\end{array}$ & $\begin{array}{c}\text { Height } \\
(\mathrm{cm})\end{array}$ & $\begin{array}{c}\text { Maximal Aerobic } \\
\text { Power } \\
(\text { MAP, W) }\end{array}$ \\
\hline Dead Space (DS) & 10 & $29.0 \pm 9.5$ & $71.8 \pm 9.1$ & $175.1 \pm 7.5$ & $231.0 \pm 31.1$
\end{tabular}

Mean \pm SD.

Table 2. Summary of three-way analysis of variance and interactions between factors.

\begin{tabular}{|c|c|c|c|c|c|c|}
\hline & $\begin{array}{c}\text { Effect } \\
\text { of } \\
\text { Exer. vs. Rest }\end{array}$ & $\begin{array}{c}\text { Effect } \\
\text { of } \\
\text { Hx vs. Nx }\end{array}$ & $\begin{array}{c}\text { Effect } \\
\text { of } \\
\mathrm{aDS}\end{array}$ & $\begin{array}{c}\text { Interaction } \\
\text { Rest / Exer. } \\
- \\
\mathrm{Nx} / \mathrm{Hx}\end{array}$ & $\begin{array}{c}\text { Interaction } \\
\text { Rest / Exer. } \\
- \\
\text { DS }\end{array}$ & $\begin{array}{c}\text { Interaction } \\
\mathrm{Nx} / \mathrm{Hx} \\
- \\
\mathrm{DS}\end{array}$ \\
\hline$\dot{\mathrm{V}} \mathrm{E}$ & $* * *$ & $* *$ & $* * *$ & ns & $*$ & ns \\
\hline $\mathrm{SpO}_{2}$ & $* * *$ & $* * *$ & ns & $* *$ & ns & ns \\
\hline $\mathrm{PETCO}_{2}$ & $* * *$ & $* * *$ & $*$ & $* *$ & ns & ns \\
\hline VT & $* * *$ & $P=0.0629$ & $* * *$ & ns & ns & $\mathrm{ns}$ \\
\hline Ttot & $* * *$ & ns & $*$ & ns & ns & ns \\
\hline $\mathrm{HR}$ & $* * *$ & $* * *$ & ns & $P=0.07$ & $\mathrm{~ns}$ & $\mathrm{~ns}$ \\
\hline$\dot{\mathrm{V} A}$ & $* * *$ & $* * *$ & ns & ns & ns & ns \\
\hline$\dot{\mathrm{V} E}$ peak power & $* * *$ & $* * *$ & $* *$ & $\mathrm{~ns}$ & $*$ & $\mathrm{~ns}$ \\
\hline$\dot{\mathrm{V} E}$ period & $* * *$ & $\mathrm{~ns}$ & $\mathrm{~ns}$ & ns & $\mathrm{ns}$ & $\mathrm{ns}$ \\
\hline
\end{tabular}

Nx: normoxia; Hx: hypoxia; Exer.: Exercise; aDS : added dead space ; VE: minute ventilation; $\mathrm{SpO}_{2}$ : pulse $\mathrm{O}_{2}$ saturation; $\mathrm{PETCO}_{2}$ : end-tidal $\mathrm{PCO}_{2}$; VT: tidal volume; Ttot: total respiration cycle time; HR: heart rate; $\dot{\mathrm{V} A}$ : alveolar ventilation. $*, P<0.05$; **, $P<0.01$; ***, $P<0.001$; ns, non-significant. 
Added dead space $=0$
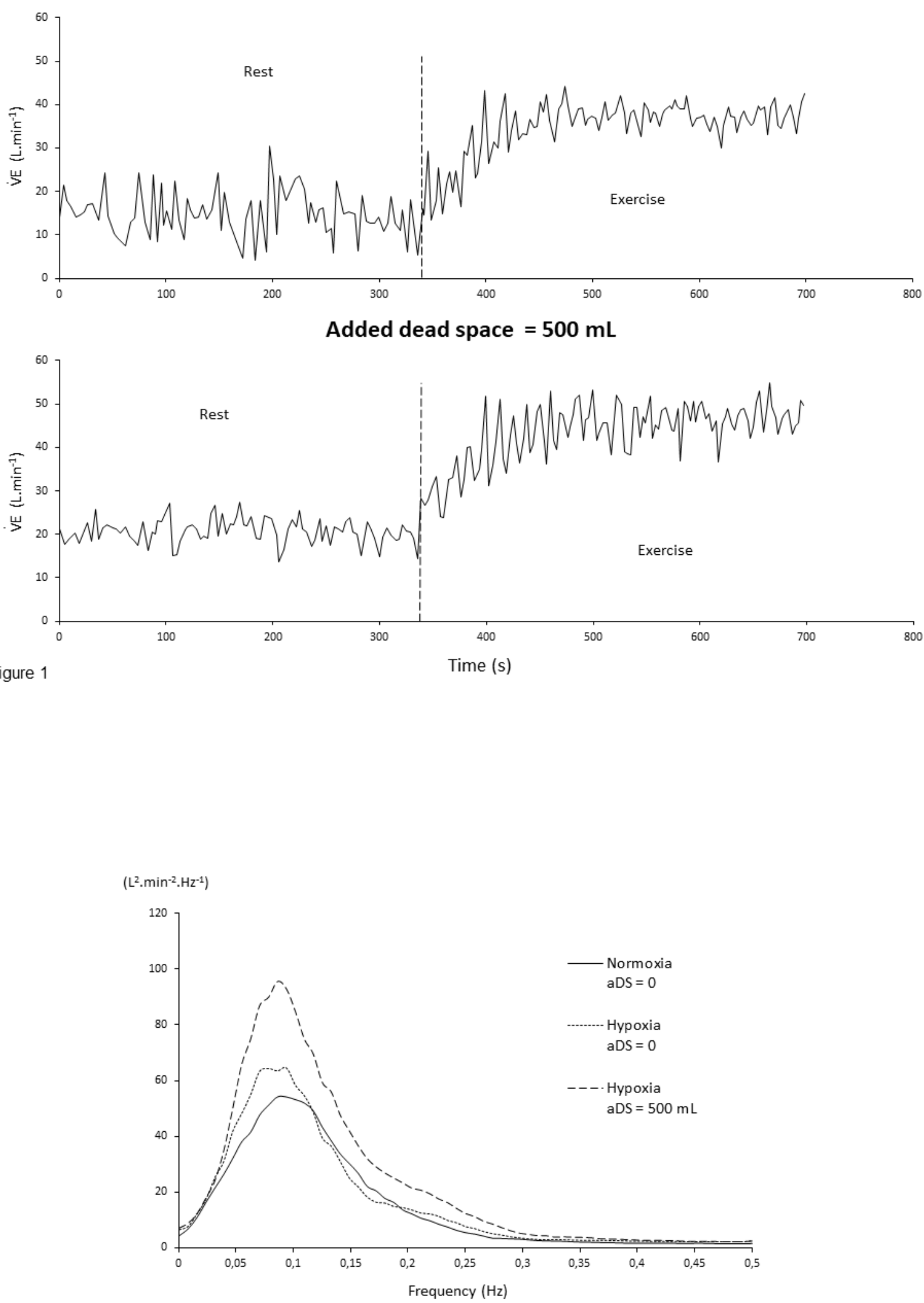

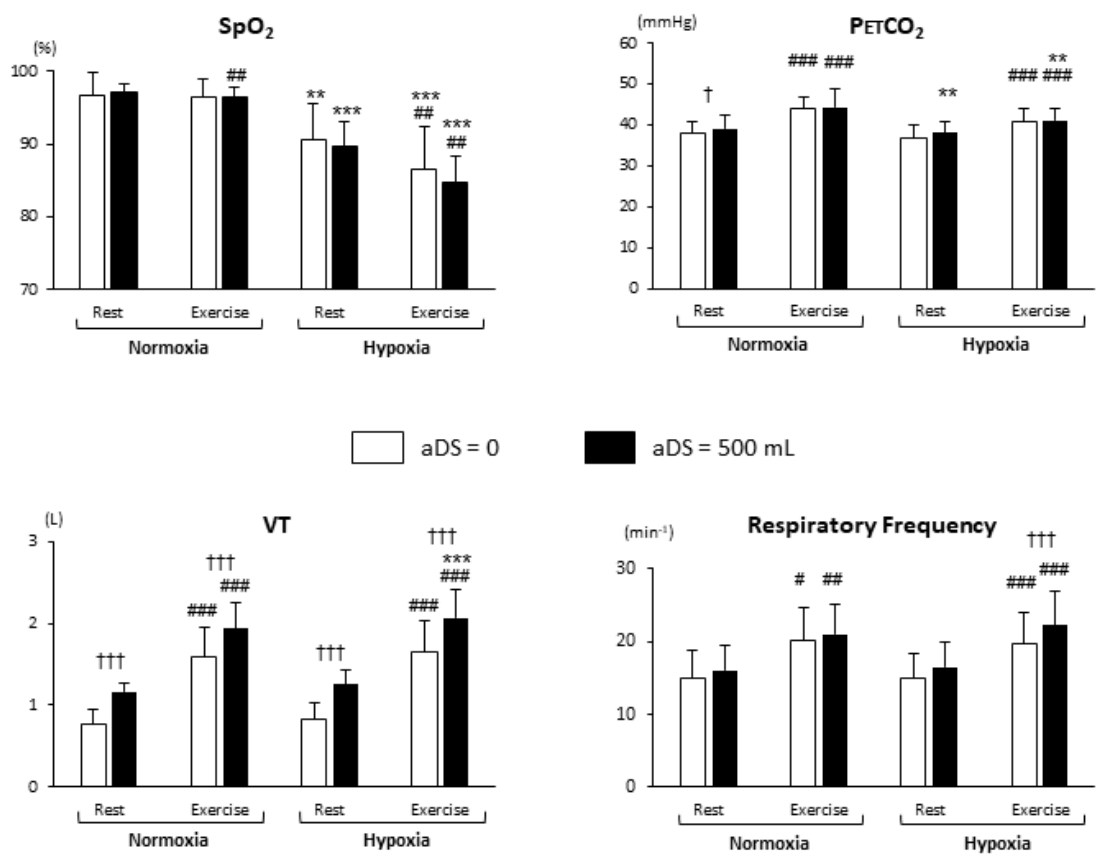

Figure 3


$\mathrm{aDS}=0$

$\mathrm{aDS}=500 \mathrm{~mL}$


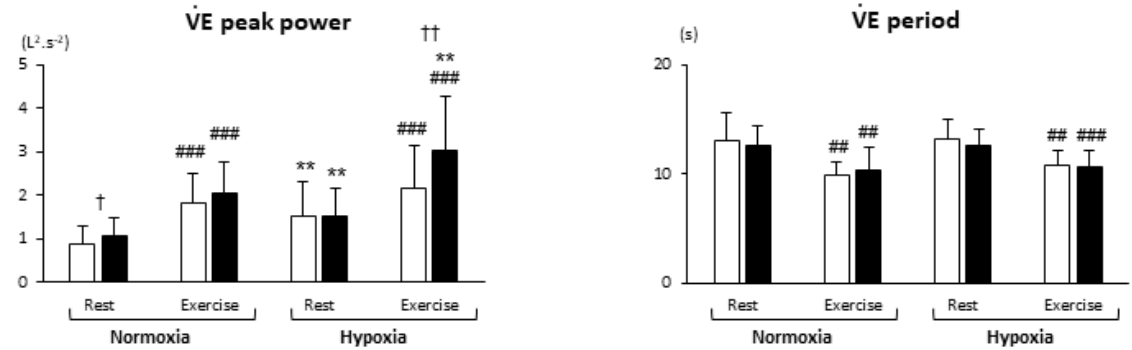

$\square \mathrm{aDS}=0$

$\mathrm{aDS}=500 \mathrm{~mL}$
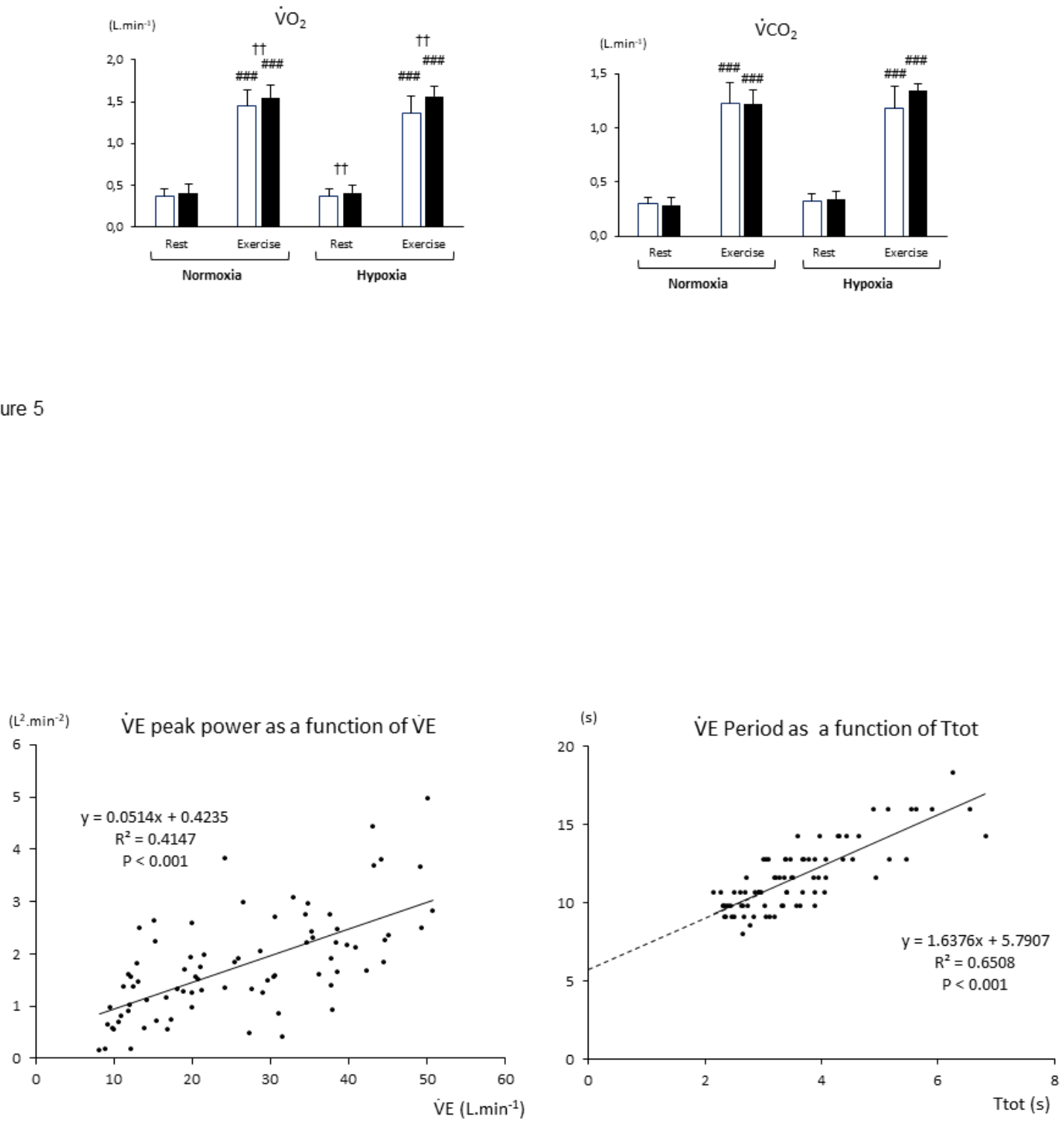